\title{
Contribuições das áreas de educação e ensino para o desenvolvimento da educomunicação
}

\author{
Ademilde Silveira Sartori \\ Doutora em Ciências da Comunicação pela Universidade de São Paulo. Professora titular do \\ Departamento de Pedagogia e do Programa de Pós-Graduação em Educação da Universidade \\ do Estado de Santa Catarina (Udesc). \\ E-mail: ademildesartori@gmail.com
Esther Bahr Pessôa Catarina (Udesc).
E-mail: estherbp@yahoo.com.br \\ Mestranda do Programa de Pós-Graduação em Educação da Universidade do Estado de Santa
}

Resumo: Neste artigo buscamos demonstrar a relevância dos programas de pós-graduação nas áreas de educação e ensino para a consolidação da educomunicação. Para isso, realizamos uma revisão integrativa de literatura, buscando teses e dissertações no Banco de Teses e Dissertações da Capes e no Banco Digital de Teses e Dissertações do Instituto Brasileiro de Informação em Ciência e Tecnologia com a palavra-chave "educomunicação". Obtivemos inicialmente 241 trabalhos, dos quais 102 foram produzidos em programas das áreas desejadas. Concluímos que esses programas representam uma parcela significativa na produção acadêmica total relacionada à educomunicação e apresentam padrões semelhantes. Defendemos, assim, que eles desempenham papel de grande importância no desenvolvimento do campo.

Palavras-chave: educomunicação; educação; ensino; revisão integrativa de literatura; pós-graduação.
Abstract: In this article we seek to demonstrate the relevance of graduate programs in the fields of Education and Teaching for the consolidation of educommunication. To do so, we conducted an integrative review of the literature, searching for theses and dissertations in the Database of Theses and Dissertations of Capes and in the Digital Bank of Theses and Dissertations of the Brazilian Institute of Information in Science and Technology using the keyword "educommunication". We initially obtained 241 works, of which 102 were produced in programs of the desired fields. We conclude that these programs represent a significant part of the total academic production related to educommunication and have similar standards. We thus defend that they play a major role in the development of the field.

Keywords: educommunication; education; teaching; integrative literature review; postgraduate studies. 
1. OROZCO GÓMEZ, Guillermo. Entre telas: novos papéis comunicativos e educativos dos cidadãos. In: APARICI, Roberto (Org.). Educomunicação: para além do 2.0 . São Paulo: Paulinas, 2014

2. SARTORI, Ademilde Silveira. Educomunicação e sua relação com a escola: a promoção de ecossistemas comunicativos e a aprendizagem distraída. Comunicação, mídia e consumo, São Paulo, v. 7, n. 19, p. 33-48, 2010.

3. SOARES, Donizete. Educomunicação: o que é isto? Gens Instituto de Educação e Cultura. São Paulo, p. 1-12, 2006, p. 1

4. AGUADED, Ignacio. Precisamos de uma revolução educomunicativa para transformar o mundo. Comunicação \& Educação, São Paulo, ano 21, n. 2, jul./dez. 2016, p. 100

5. HOPPE, Bárbara Chiodini Axt. Práticas educomunicativas no contexto escolar: obstáculos e perspectivas. Dissertação (mestrado em extensão rural), Universidade Federal de Santa Maria, Rio Grande do Sul, 2016, p. 59.

6. CORNÉLIO, Camila Gallindo. Educomunicação na escola, faz sentido?: Projetos educomunicativos de uma escola de referência em ensino médio a partir do que falam os estudantes. Dissertação (mestrado em educação), Universidade Federal de Pernambuco, Pernambuco, 2016.

\section{EDUCAÇÃO E COMUNICAÇÃO: RUMO À EDUCOMUNICAÇÃO}

Em uma sociedade cada vez mais permeada pelas tecnologias da informação e comunicação (TIC), é inegável que essas tecnologias influenciam cada vez mais os diversos aspectos da vida humana. Orozco Gómez ${ }^{1}$ afirma que a interação com as telas não é mais algo que se faça esporadicamente, para buscar informação ou entretenimento, mas uma necessidade.

Ainda que nem todos tenham acesso a essas transformações na mesma medida, a grande maioria da população é afetada pelos meios de comunicação em algum nível. A escola e o universo da educação também são atingidos pelas mudanças introduzidas pelas novas tecnologias. Sartori ${ }^{2}$ afirma que os meios de comunicação somam às tradicionais dificuldades da escola um novo desafio, ao descentralizarem as formas de transmissão e circulação do saber. Somam-se ainda os desafios gerados pelos diferentes níveis de acesso aos ecossistemas comunicativos e informacionais.

Uma escola que pretenda responder às demandas trazidas por essa nova realidade não pode se furtar às questões relacionadas à comunicação e às mídias. Diante das indagações e mudanças provocadas pelo desenvolvimento das TIC, a educomunicação tem surgido como possível resposta para algumas inquietações. Tal processo pode ser notado através do número crescente de teses e dissertações produzidas em programas de pós-graduação em educação e outros semelhantes que versam sobre a educomunicação.

O termo "educomunicação" é definido de forma diversa por diferentes autores, refletindo a polissemia do campo. Neste trabalho, compreendemos que

Quando falamos em educomunicação, estamos nos referindo a um campo de pesquisa, de reflexão e de intervenção social, cujos objetivos, conteúdos e metodologia são essencialmente diferentes tanto da educação escolar quanto da comunicação social ${ }^{3}$.

Aguaded ressalta a relevância do campo, destacando seu caráter de convergência entre educação e comunicação, ambas áreas fundamentais para o desenvolvimento dos povos, especialmente em termos sociais e culturais. Afirma, entretanto, que

Por outro lado, é um campo de estudos muito novo, que demanda pesquisa constante porque suas premissas ainda estão em construção. Necessitamos de pesquisa rigorosa e sistemática sobre as novas tendências da comunicação, especialmente quanto aos usos da população para a construção de propostas reais que permitam às pessoas conviver com a mídia de uma forma integral ${ }^{4}$.

Hoppe afirma que "as práticas e teorias educomunicativas aliadas às dinâmicas de ensino-aprendizagem podem trazer novas perspectivas para educadores e educandos" . Para Cornélio ${ }^{6}$, a implantação de projetos educomunicativos na escola está diretamente relacionada à criação de espaços escolares que façam sentido para os estudantes. 
Movidas pela inquietação a respeito do real papel da educomunicação perante os desafios da educação formal, nos propomos a analisar como está se dando o processo de incorporação dos estudos da educomunicação nas áreas de educação e ensino, a partir das produções realizadas nos programas de pós-graduação de nosso país. Definimos como objetivo deste trabalho mapear a produção acadêmica brasileira nas áreas de educação e ensino que trata da educomunicação, descrevendo quantitativamente a pesquisa disponível em duas bases digitais de teses e dissertações. Buscamos, assim, verificar a existência de semelhanças entre o total de trabalhos encontrados e a produção realizada especificamente nas áreas de educação e ensino. Portanto, realizamos um trabalho de caráter bibliográfico. Uma vez que, ao iniciar a pesquisa, não era possível precisar que tipos de produções encontraríamos, optamos por uma revisão integrativa, que permite analisar trabalhos feitos com diferentes metodologias ${ }^{7}$, de forma que seria possível incluir todas as teses e dissertações encontradas. Descrevemos o percurso metodológico seguido para a pesquisa na próxima seção.

\section{PERCURSO METODOLÓGICO}

As revisões de literatura são de grande importância para o desenvolvimento científico, pois permitem identificar lacunas e oportunidades em um campo de conhecimento específico, bem como servem de base para o surgimento de novas teorias $^{8}$. Os trabalhos de revisão "são uma forma de pesquisa que utilizam de fontes de informações bibliográficas ou eletrônicas para obtenção de resultados de pesquisas de outros autores, com o objetivo de fundamentar teoricamente um determinado objetivo" .

Existem dois tipos principais de revisão de literatura: o narrativo e o sistemático. Ambos têm caráter retrospectivo e observacional, mas se diferenciam especialmente pelos métodos empregados para evitar erros e vieses ${ }^{10}$. As revisões narrativas, também conhecidas como tradicionais, caracterizam-se pela falta de uma metodologia científica estabelecida para sua elaboração. Geralmente possuem natureza opinativa, que seleciona livros e artigos a serem utilizados conforme a intenção. Revisões narrativas não informam a metodologia utilizada para a busca de referências, nem os critérios adotados para seleção e avaliação dos trabalhos. Uma vez que as informações estão sujeitas a um forte viés de seleção, considera-se que essas revisões possuem força de evidência científica fraca $^{11,12}$.

Já as revisões bibliográficas sistemáticas são conduzidas tendo como objetivo responder a uma pergunta específica e possuem métodos bem definidos para busca, seleção e avaliação dos trabalhos ${ }^{13}$. Segundo Rother ${ }^{14}$, "os trabalhos de Revisão Sistemática são considerados trabalhos originais, pois, além de utilizar como fonte, dados da literatura sobre determinado tema, são elaborados com rigor metodológico", o que possibilita atribuir a essas revisões força de evidência científica.
7. SOARES, Cassia Baldini et al. Revisão integrativa: conceitos e métodos utilizados na enfermagem. Revista da Escola de Enfermagem da USP, São Paulo, v. 48, n. 2, p. 335-345, 2014.

8. BOTELHO, Larissa Lira Roedel; CUNHA, Cristiano Castro de Almeida; MACEDO, Marcelo. O método da revisão integrativa nos estudos organizacionais. Gestão e sociedade, Belo Horizonte, v. 5, n. 11, p. 121-136, maio/ago. 2011.

9. ROTHER, Edna Terezinha. Revisão sistemática $X$ revisão narrativa. Acta Paulista de Enfermagem, São Paulo, v. 20, n. 2, p. 5-6, abr./jun. 2007, p. 5.

10. COOK, Debora J.; MULROW, Cynthia D.; RAYNES, R. Brian. Systematic reviews: synthesis of best evidence for clinical decisions. Annals of Internal Medicine, Philadelphia, v. 126, n. 5, p. 376-380, mar. 1997.

11. BERNARDO, Wanderley Marques; NOBRE, Moacyr Roberto Cuce; JATENE, Fábio Biscegli. A prática clínica baseada em evidências. Parte II buscando as evidências em fontes de informação. Revista da Associação Médica Brasileira, São Paulo, v. 50, n. 1, p. $104-$ 108, 2004.

12. ROTHER, Edna Terezinha. Revisão sistemática $X$ revisão narrativa, op. cit., p. 6.

13. COOK, Debora J.; MULROW, Cynthia D.; RAYNES, R. Brian. Systematic reviews, op. cit.

14. ROTHER, Edna Terezinha. Revisão sistemática $X$ revisão narrativa, op. cit., p. 5. 
15. URSI, Elisabeth Silva. Prevenção de lesões de pele no perioperatório: revisão integrativa da literatura. Dissertação (mestrado), Escola de Enfermagem de Ribeirão Preto, Universidade de São Paulo, Ribeirão Preto, 2005, p. 36

16. MENDES, Karina Dall Sasso; SILVEIRA, Renata Cristina de Campos Pereira; GALVÃO, Cristina Maria. Revisão integrativa: método de pesquisa para a incorporação de evidências na saúde e na enfermagem. Texto \& Contexto Enfermagem, Florianópolis, v. 17, n. 4, p. 758-764, out./dez. 2008, p. 758.

17. WHITEMORE, Robin; KNAFL, Kathleen. The integrative review: updated methodology. Journal of Advanced Nursing. v. 52, n. 5 , p. $546-553,2005$

18. Idem, ibidem

19. MENDES, Karina Dall Sasso; SILVEIRA, Renata Cristina de Campos Pereira; GALVÃO, Cristina Maria. Revisão integrativa, op. cit., p. 759.

20. BOTELHO, Larissa Lira Roedel; CUNHA, Cristiano Castro de Almeida; MACEDO, Marcelo. O método da revisão integrativa nos estudos organizacionais, op. cit.
Dentro das revisões sistemáticas de literatura se encaixam quatro subtipos, a saber: revisão sistemática, metanálise, revisão qualitativa e revisão integrativa. A última é "um método em que pesquisas anteriores são sumarizadas e conclusões são estabelecidas considerando o delineamento das pesquisas avaliadas, a qual possibilita síntese e análise do conhecimento científico já produzido do tema investigado"15. Uma revisão integrativa pode servir a diferentes finalidades, entre as quais se destacam: definir conceitos, revisar teorias, realizar análise metodológica de trabalhos relacionados a um mesmo tópico ${ }^{16}$. As revisões integrativas constituem o tipo mais amplo e abrangente de revisão sistemática ${ }^{17}$.

No intuito de organizar a elaboração das revisões integrativas, autores têm proposto etapas nas quais se desenvolve uma revisão integrativa. Com base nos processos determinados por Whitemore e Knalf ${ }^{18}$, por Mendes, Silveira e Galvão ${ }^{19}$ e por Botelho, Cunha e Macedo ${ }^{20}$, estabelecemos para a realização desta revisão integrativa dez etapas a serem seguidas, buscando maior detalhamento das ações: 1. escolha do tema e definição da questão de pesquisa; 2. definição das estratégias de busca (palavras-chave, bancos de dados, operadores e técnicas de busca, estratégias complementares); 3. definição dos critérios de inclusão e exclusão; 4. busca nas bases de dados e seleção dos estudos, segundo os critérios definidos; 5. construção do instrumento para seleção das informações; 6 . seleção e organização das informações extraídas dos estudos, utilizando o instrumento construído; 7. categorização dos estudos segundo critérios de semelhança, diferença, relações entre eles; 8. análise dos dados; 9. interpretação dos resultados; 10. construção do documento de apresentação da revisão.

As decisões relacionadas ao processo de execução desta revisão foram tomadas sempre levando em consideração o objetivo proposto, bem como as disponibilidades de tempo e de acesso à informação. Com o objetivo de manter a busca a mais ampla possível, definimos como palavra-chave o termo "educomunicação", sendo aceitas também variações dessa palavra, como as formas adjetivadas "educomunicativa" ou "educomunicativo", no singular e no plural. Uma vez que nosso objetivo se resume a mapear o desenvolvimento do campo da educomunicação em específico, optamos por não incluir pesquisas que se refiram, por exemplo, à mídia-educação, educação para os meios, entre outros assuntos. Interessam-nos apenas as que se inserem diretamente no campo da educomunicação.

As bases de dados foram selecionadas levando em consideração sua abrangência e relevância. Como principal fonte de dados, utilizamos o Banco Digital de Teses e Dissertações da Coordenação de Aperfeiçoamento de Pessoal de Nível Superior (Capes), pois nele se encontra organizada parte significativa das teses e dissertações produzidas no Brasil. O sistema permite a utilização de alguns operadores, que podem servir para garantir a abrangência da busca. Nesta pesquisa, utilizamos o asterisco (*) no final da palavra, recurso que permite a busca por variações de sufixo e que também pode ser usado no meio do termo. Preocupadas em garantir a abrangência da pesquisa, realizamos uma busca auxiliar na Biblioteca Digital Brasileira de Teses e Dissertações (BDTD), mantida pelo Instituto Brasileiro de Informação em Ciência e Tecnologia (Ibict). 
O Quadro 1 apresenta de forma resumida as principais informações relacionadas à revisão.

\section{Quadro 1: Descrição das etapas da pesquisa}

\begin{tabular}{|c|c|}
\hline ETAPA & DESCRIÇÃO \\
\hline Tema & $\begin{array}{l}\text { Produção acadêmica sobre educomunicação nas áreas de } \\
\text { educação e ensino }\end{array}$ \\
\hline Questão de pesquisa & $\begin{array}{l}\text { De que forma a educomunicação aparece na produção } \\
\text { científica e acadêmica nas áreas de educação e ensino } \\
\text { no Brasil? }\end{array}$ \\
\hline \multicolumn{2}{|l|}{ Estratégias de busca } \\
\hline Palavras-chave & Educomunica* \\
\hline Bancos de dados & BDTD, Portal Capes \\
\hline Operadores e técnicas de busca & * para abranger diferentes sufixos da palavra. \\
\hline Estratégias complementares & $\begin{array}{l}\text { Solicitação das informações diretamente aos autores, } \\
\text { programas de pós-graduação ou bibliotecas, quando não } \\
\text { disponíveis na internet. }\end{array}$ \\
\hline Critérios de inclusão & $\begin{array}{l}\text { 1. possuir a palavra-chave definida para a busca no título, } \\
\text { no resumo ou nas palavras-chave; } \\
\text { 2. pertencer a programas de pós-graduação nas áreas de } \\
\text { educação, ensino ou similar. }\end{array}$ \\
\hline Critérios de exclusão & $\begin{array}{l}\text { Indisponibilidade das informações necessárias (não estão } \\
\text { disponíveis na internet e não foram encontradas através de } \\
\text { recursos complementares, como contato direto com o autor). }\end{array}$ \\
\hline Busca e seleção dos estudos & $\begin{array}{l}\text { Realizada entre os meses de março e abril de 2017, através } \\
\text { da internet. }\end{array}$ \\
\hline $\begin{array}{l}\text { Instrumentos para seleção das } \\
\text { informações }\end{array}$ & $\begin{array}{l}\text { Tabela } 1 \text { para extração e organização das seguintes } \\
\text { informações: autor, orientador, ano, programa de pós- } \\
\text { graduação, universidade, título, resumo e palavras-chave } \\
\text { (utilizada para todos os trabalhos que se encaixaram nos } \\
\text { critérios de inclusão } 1 \text { e 2). }\end{array}$ \\
\hline $\begin{array}{l}\text { Seleção e organização das } \\
\text { informações extraídas dos estudos }\end{array}$ & Análise quantitativa das produções a partir da Tabela 1. \\
\hline Categorização dos estudos & Não incluída neste artigo. \\
\hline Análise dos dados & Não incluída neste artigo. \\
\hline Interpretação dos resultados & Não incluída neste artigo. \\
\hline $\begin{array}{l}\text { Documento de apresentação da } \\
\text { revisão }\end{array}$ & $\begin{array}{l}\text { Artigo contendo os resultados parciais. } \\
\text { Dissertação contendo os resultados finais. }\end{array}$ \\
\hline
\end{tabular}

Fonte: Produção das autoras, 2017

\section{SEMELHANÇAS E DIFERENÇAS NA PRODUÇÃO}

Conforme diretrizes expressas na seção anterior, inicialmente realizamos uma busca no Banco de Teses e Dissertações da Capes, encontrando 276 trabalhos. 
Para garantir a abrangência da pesquisa, realizamos uma busca auxiliar no Banco Digital de Teses e Dissertações (BDTD, encontrando 146 resultados. Após excluir manualmente as repetições, ficamos com 143 trabalhos. Destes, treze eram resultados inéditos, que não apareceram na pesquisa realizada no Banco da Capes.

Obtivemos, assim, 290 teses e dissertações como retorno inicial. Aplicamos a elas nosso primeiro critério de inclusão: nos interessavam apenas as teses e dissertações cujo termo "educomunicação" ou similar aparecesse no título, nas palavras-chave ou no resumo. Ficamos, assim, com um total de 241 trabalhos, dos quais 210 dissertações e 31 teses.

As teses e dissertações foram produzidas em diversos programas de pós-graduação, como pode ser visto no Quadro 2, destacando assim a amplitude e interdisciplinaridade do campo. Há trabalhos provenientes de áreas tão diversas quanto o direito, a arquitetura e a engenharia. Em geral, no entanto, destacam-se programas de pós-graduação relacionados a educação, tecnologias ou meio ambiente. Os programas com maior número de trabalhos são: em educação, com 62 dissertações e 8 teses; em ciências da comunicação, com 34 dissertações e 16 teses; e em comunicação, com 23 dissertações e 1 tese.

\section{Quadro 2: Produção de teses e dissertações por programa de pós-graduação}

\begin{tabular}{|c|c|c|c|c|c|}
\hline $\begin{array}{l}\text { PROGRAMA DE } \\
\text { PÓS-GRADUAÇÃO EM }\end{array}$ & M & D & $\begin{array}{l}\text { PROGRAMA DE } \\
\text { PÓS-GRADUAÇÃO EM }\end{array}$ & M & D \\
\hline Educação & 61 & 8 & $\begin{array}{l}\text { Desenvolvimento regional } \\
\text { (profissional) }\end{array}$ & 1 & \\
\hline Ciências da comunicação & 34 & 16 & Difusão do conhecimento & & 1 \\
\hline Comunicação & 23 & 1 & Direito & 1 & \\
\hline Comunicação social & 7 & 1 & Divulgação científica e cultural & 1 & \\
\hline Ciências & 4 & & Educação agrícola & 1 & \\
\hline Comunicação e cultura & 4 & & Educação ambiental & 1 & \\
\hline Educação matemática e tecnológica & 4 & & Educação e diversidade (profissional) & 1 & \\
\hline Estudos da linguagem & 3 & 1 & Educação escolar & 1 & \\
\hline Desenvolvimento e meio ambiente & 3 & & Educação nas ciências & 1 & \\
\hline Ensino de ciências (profissional) & 3 & & Educação sexual & 1 & \\
\hline $\begin{array}{l}\text { Desenvolvimento regional e meio } \\
\text { ambiente }\end{array}$ & 2 & & Engenharia ambiental & & 1 \\
\hline Desenvolvimento sustentável & 2 & & Engenharia de produção & 1 & \\
\hline Educação brasileira & 1 & 1 & Ensino de ciências & 1 & \\
\hline Educação científica e tecnológica & 1 & 1 & Ensino de ciências e matemática & 1 & \\
\hline Educação, cultura e comunicação & 2 & & $\begin{array}{l}\text { Ensino de ciências na Amazônia } \\
\text { (profissional) }\end{array}$ & 1 & \\
\hline $\begin{array}{l}\text { Educação, cultura e territórios } \\
\text { semiáridos }\end{array}$ & 2 & & $\begin{array}{l}\text { Ensino de ciências naturais e } \\
\text { matemática }\end{array}$ & 1 & \\
\hline
\end{tabular}


Contribuições das áreas de educação e ensino para o desenvolvimento da educomunicação

- Ademilde Silveira Sartori e Esther Bahr Pessôa

\begin{tabular}{|c|c|c|c|}
\hline Extensão Rural & 2 & $\begin{array}{l}\text { Ensino em ciências da saúde } \\
\text { (profissional) }\end{array}$ & 1 \\
\hline $\begin{array}{l}\text { Gestão em organizações } \\
\text { aprendentes (profissional) }\end{array}$ & 2 & $\begin{array}{l}\text { Ensino, filosofia e história das } \\
\text { ciências }\end{array}$ & 1 \\
\hline Psicologia social & 2 & $\begin{array}{l}\text { Ensino, história e filosofia das } \\
\text { ciências e matemática }\end{array}$ & 1 \\
\hline Tecnologia & 2 & Estudos da mídia & 1 \\
\hline $\begin{array}{l}\text { Tecnologias, comunicação e } \\
\text { educação }\end{array}$ & 2 & Gestão de recursos naturais & 1 \\
\hline Ambiente e desenvolvimento & 1 & Indústria criativa (profissional) & 1 \\
\hline Arquitetura e urbanismo & 1 & Integração da América Latina & 1 \\
\hline Artes visuais & 1 & Letras (profissional) & 1 \\
\hline $\begin{array}{l}\text { Ciências do ambiente e } \\
\text { sustentabilidade na Amazônia }\end{array}$ & 1 & Meio ambiente e desenvolvimento & 1 \\
\hline $\begin{array}{l}\text { Ciências da saúde e do meio } \\
\text { ambiente (profissional) }\end{array}$ & 1 & Mídia e cotidiano & 1 \\
\hline Ciência e tecnologia ambiental & 1 & Mídia e cultura & 1 \\
\hline $\begin{array}{l}\text { Ciências humanas, sociais e da } \\
\text { natureza }\end{array}$ & 1 & Políticas sociais & 1 \\
\hline Ciências sociais & 1 & Saúde pública & 1 \\
\hline Ciência, tecnologia e educação & 1 & Sociedade e cultura na Amazônia & 1 \\
\hline Comunicação e linguagens & 1 & $\begin{array}{l}\text { Tecnologias da inteligência e design } \\
\text { digital }\end{array}$ & 1 \\
\hline Comunicação e semiótica & 1 & $\begin{array}{l}\text { Tecnologias educacionais em rede } \\
\text { (profissional) }\end{array}$ & 1 \\
\hline Comunicação e territorialidades & 1 & $\begin{array}{l}\text { Tecnologia e gestão em educação } \\
\text { a distância }\end{array}$ & 1 \\
\hline Comunicação midiática & 1 & & \\
\hline
\end{tabular}

Fonte: Produção das autoras, 2017.

Realizaremos, a seguir, uma análise quantitativa dos trabalhos encontrados. Também compararemos as produções sobre educomunicação em geral e os trabalhos apenas nas áreas de educação e ensino, buscando verificar se ambos seguem os mesmos padrões. Sendo assim, aplicamos o segundo critério de inclusão, conforme Quadro 1 - produção em programas de pós-graduação de áreas diretamente relacionadas à educação ou ao ensino -, obtendo um total de 92 dissertações e 10 teses.

Os trabalhos incluídos nesta pesquisa são provenientes de 69 universidades (Quadro 3). A Universidade de São Paulo (USP) apresenta o maior número de trabalhos, 64, seguida pela Universidade Federal do Paraná (UFPR), com 18, e pela Universidade do Estado de Santa Catarina (Udesc), com 10. Do total de universidades citadas, 35 contam com apenas uma tese ou dissertação constante na pesquisa.

Já as 102 produções nas áreas de educação e ensino são provenientes de 46 universidades. A Udesc e a Universidade Federal do Paraná (UFPR) são as instituições com maior número de trabalhos, a saber, 10 cada uma. A USP tem 
a segunda maior quantidade, 7 produções, seguida pela Universidade Federal de Pernambuco (UFPE), com 23 instituições possuem apenas um trabalho cada.

\section{Quadro 3: Produção de teses e dissertações por universidade com produção acima de um trabalho}

\begin{tabular}{|c|c|c|c|}
\hline & & G & $E$ \\
\hline Universidade de São Paulo (USP) & est & 64 & 7 \\
\hline Universidade Federal do Paraná (UFPR) & fed & 18 & 10 \\
\hline Universidade do Estado de Santa Catarina (Udesc) & est & 10 & 10 \\
\hline Universidade Metodista de São Paulo & priv & 8 & 2 \\
\hline Universidade de Brasília (UnB) & fed & 6 & 3 \\
\hline Universidade Federal de Pernambuco (UFPE) & fed & 6 & 6 \\
\hline Universidade Federal de Santa Maria (UFSM) & fed & 6 & 1 \\
\hline Pontifícia Universidade Católica de São Paulo (PUC-SP) & priv & 5 & 2 \\
\hline Universidade Federal da Paraíba (UFPB) & fed & 5 & 2 \\
\hline Universidade Federal de Mato Grosso (UFMT) & fed & 5 & 4 \\
\hline Universidade Federal do Amazonas (Ufam) & fed & 5 & 2 \\
\hline Universidade do Vale do Rio dos Sinos (Unisinos) & priv & 4 & \\
\hline Universidade do Estado da Bahia & est & 4 & 3 \\
\hline Universidade Estadual Paulista Júlio de Mesquita Filho (Unesp) & est & 4 & 3 \\
\hline Universidades Federal de Santa Catarina (UFSC) & fed & 4 & 2 \\
\hline Universidade Tecnológica Federal do Paraná (UTFPR) & fed & 4 & \\
\hline Universidade de Marília & priv & 3 & \\
\hline Universidade de Sorocaba & priv & 3 & \\
\hline Universidade do Oeste Paulista & priv & 3 & 3 \\
\hline Universidade Estadual de Campinas (Unicamp) & est & 3 & 2 \\
\hline Universidade Federal da Bahia (Ufba) & fed & 3 & 2 \\
\hline Universidade Federal de Goiás (UFG) & fed & 3 & 1 \\
\hline Universidade Federal de Uberlândia (UFU) & fed & 3 & 2 \\
\hline Universidade Federal do Ceará (UFC) & fed & 3 & 3 \\
\hline Universidade Federal do Espírito Santo (Ufes) & fed & 3 & 2 \\
\hline Universidade Federal do Rio Grande do Norte (UFRN) & fed & 3 & 1 \\
\hline Universidade Paulista & priv & 3 & \\
\hline Universidade de Uberaba & priv & 2 & 2 \\
\hline Universidade do Estado de Minas Gerais (Uemg) & est & 2 & 2 \\
\hline Universidade do Estado do Rio de Janeiro (Uerj) & est & 2 & 2 \\
\hline Universidade do Estado do Rio Grande do Norte & est & 2 & 1 \\
\hline Universidade Estadual de Londrina (UEL) & est & 2 & 1 \\
\hline Universidade Federal de Juiz de Fora (UFJF) & fed & 2 & \\
\hline Universidade Federal do Rio de Janeiro (UFRJ) & fed & 2 & \\
\hline Universidade Nove de Julho (Uninove) & priv & 2 & 2 \\
\hline
\end{tabular}


Não é surpresa que a USP tenha o maior número de produções, uma vez que a Escola de Comunicações e Artes (ECA) pode ser considerada o principal polo de educomunicação no Brasil. No entanto, sua produção acontece prioritariamente na área de ciências da comunicação, por isso, não admira que o número de produções decresça consideravelmente quando reduzimos a busca apenas para trabalhos nas áreas de educação e ensino. No entanto, as três universidades com maior número de produções são as mesmas em ambos os casos. Dentre as instituições com mais de uma produção sobre o tema, sete possuem todos esses trabalhos provenientes de programas nas áreas de educação ou ensino. São estas: Udesc, UFPE, Universidade do Oeste Paulista, UFC, Uemg, Uerj e Uninove.

Em ambos os recortes descritos, pode-se notar predominância de universidades públicas sobre as privadas, conforme Gráfico 1. No total das produções, 196 são provenientes de universidades públicas, sendo 99 de estaduais, 96 de federais e uma de universidade municipal. Os 45 trabalhos restantes foram produzidos em universidades privadas. Quando reduzimos o foco apenas para os trabalhos nas áreas de educação e ensino, temos 51 produções de universidades federais e 35 de universidades estaduais. As universidades privadas respondem pelos 16 trabalhos restantes.

Gráfico 1: Produção de teses e dissertações por tipo de universidade

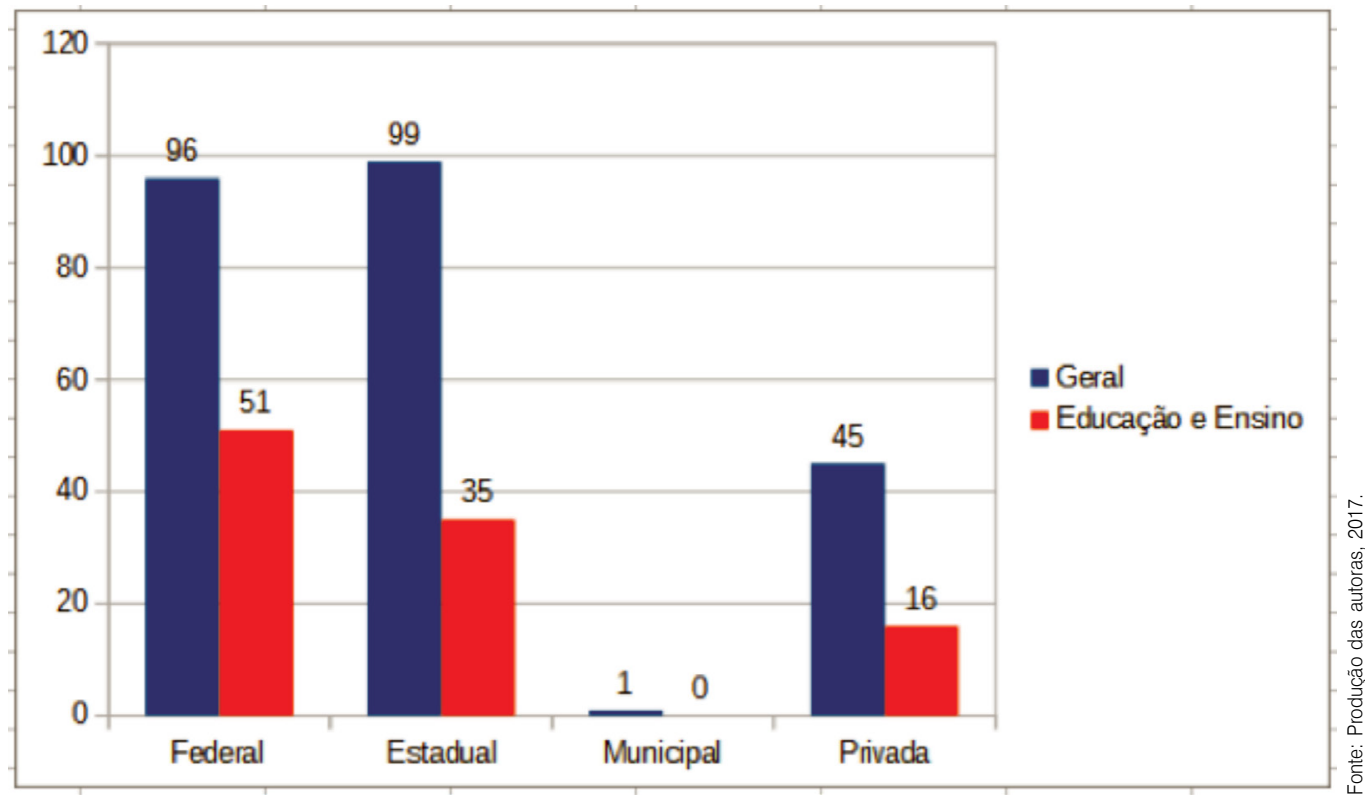

A região geográfica de maior destaque é o Sudeste, com 128 dissertações e teses, das quais 104 são do estado de São Paulo, 13 de Minas Gerias, 8 do Rio de Janeiro e 3 do Espírito Santo. Em seguida, temos a região Sul, com 59 trabalhos, dos quais 28 são do Paraná, 17 do Rio Grande do Sul e 14 de Santa Catarina. Em terceiro lugar, a região Nordeste, com 33 trabalhos, sendo 8 da 
Bahia, 7 de Pernambuco, 6 do Rio Grande do Norte, 5 da Paraíba, 4 do Ceará, 2 de Sergipe e 1 de Alagoas. O Centro-Oeste vem a seguir, com um total de 15 produções, a saber, 7 do Distrito Federal, 5 do Mato Grosso e 3 de Goiás. Finalmente, o Amazonas é o único estado da região Norte com trabalhos pertencentes ao escopo da nossa pesquisa; possui 6 produções, que representam também o total da região.

Restringindo a busca apenas para a área de educação e ensino, a região Sudeste tem o maior número de produções, 38, sendo 24 do estado de São Paulo, 8 de Minas Gerais, 4 do Rio de Janeiro e 2 do Espírito Santo. Em seguida, temos a região Sul, com 31 trabalhos, sendo 14 do Paraná, 12 de Santa Catarina e 5 do Rio Grande do Sul. O Nordeste, em terceiro lugar, conta com 22 trabalhos: 7 de Pernambuco, 5 da Bahia, 4 do Ceará, 2 do Rio Grande do Norte, 2 da Paraíba, 1 de Sergipe e 1 de Alagoas. A região Centro-Oeste possui 8 produções, sendo 4 do Mato Grosso, 3 do Distrito Federal e 1 de Goiás. Finalmente, o Amazonas responde pelos 3 trabalhos da região Norte. Essa distribuição pode ser vista no Gráfico 2.

Gráfico 2: Produção de teses e dissertações por região geográfica

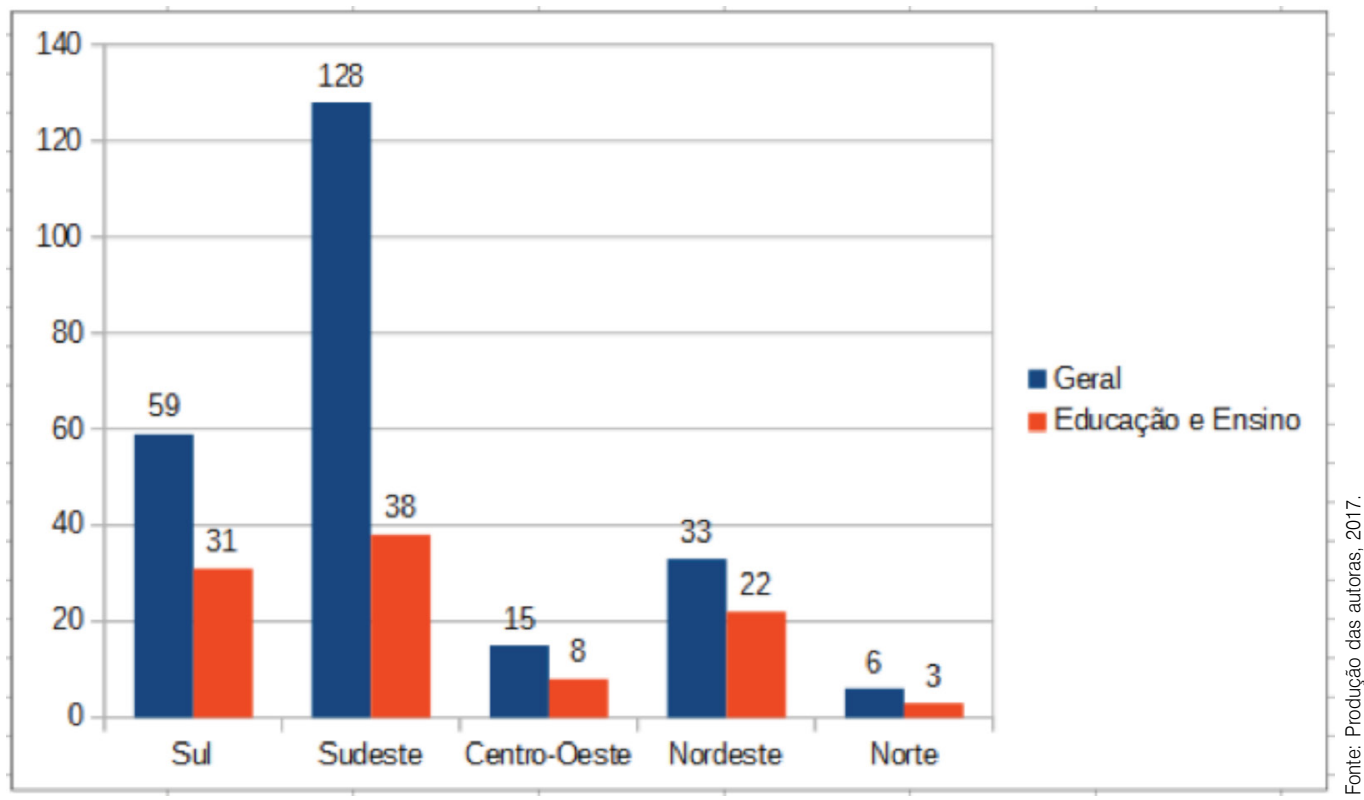

Apesar de a quantidade de produções ser menor, as regiões de destaque são as mesmas no que diz respeito tanto à produção geral quanto às especificamente voltadas para educação e ensino. No entanto, a diferença entre a primeira e a segunda colocadas diminui sensivelmente. Essa diminuição se deve ao fato de a região Sudeste possuir um número muito significativo de programas na área de comunicação e ciências da comunicação, que são excluídos no segundo caso. Nas demais regiões, no entanto, os trabalhos nas áreas de educação e ensino correspondem a, pelo menos, $50 \%$ da produção total da região. 
Em relação à distribuição no tempo (Gráfico 3), a produção ganha maior volume a partir de 2009, aproximadamente. É preciso lembrar, entretanto, que o ano levado em consideração é o de defesa da tese ou dissertação, que levou de dois a quatro anos para ser elaborada. Com base nisso, é possível inferir um aumento no interesse por essa temática a partir de 2009, tendência seguida tanto pela produção total quanto pela produção em programas de educação e de ensino. Em ambos os casos, o ano com maior número de teses e dissertações defendidas é 2016, com 35 trabalhos no total, dos quais 16 nas áreas citadas.

Gráfico 3: Produção de teses e dissertações por ano

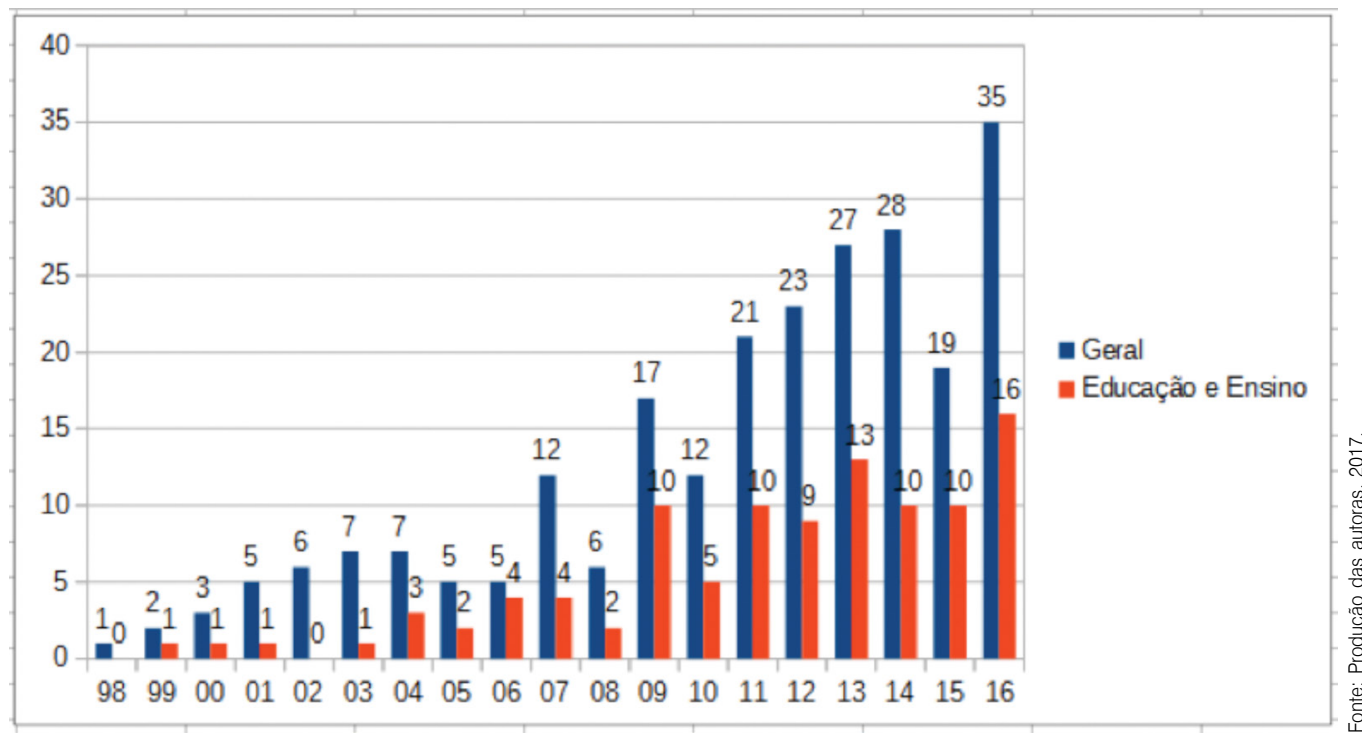

De forma geral, a tendência ao crescimento no número de pesquisas é constante em ambos os casos, demonstrando o aumento do interesse pela educomunicação com o passar do tempo.

Ao analisarmos as relações entre a produção anual total de cada estado, alguns aspectos se fazem notar. São Paulo, além de deter a maior produção, tem também a distribuição mais ampla no tempo: desde 1998, em todos os anos há trabalhos provenientes desse estado. Ele é o único com produção significativa antes de 2005, o que também se aplica à região Sudeste. Ainda assim, a produção dessa região aumenta em anos mais recentes. O Centro-Oeste é a última região a iniciar publicações nessa área, sendo o trabalho mais antigo publicado em 2004. À medida que avançamos no tempo, a produção não se torna apenas maior, mas também geograficamente mais abrangente. Dentre os estados representados nesta pesquisa, Alagoas é o único que não possui nenhum trabalho na última década, uma vez que sua única produção data de 2006. Em relação aos programas nas áreas de educação e ensino, é possível notar algumas diferenças, como o fato de o trabalho mais antigo ser proveniente do Nordeste, seguido pelo Sudeste. Também nesta região, no entanto, com o passar 
do tempo, a produção passa a se distribuir de forma mais ampla, abrangendo um número maior de estados.

\section{CONSIDERAÇÕES FINAIS}

Acreditamos que compreender melhor a produção acadêmica nas áreas de educação e ensino é importante para consolidar a educomunicação como campo de estudo. A produção nas áreas citadas segue, em linhas gerais, os mesmos padrões da produção total relacionada a esse campo, conforme demonstrado pelos resultados desta pesquisa. Ambas apresentam tendência geral de crescimento, apresentando maior volume e consistência a partir do ano de 2009, aproximadamente.

Das 241 teses e dissertações que tratam da educomunicação, 102 foram produzidas em programas das áreas de nosso interesse, representando $40 \%$ do total. Semelhantemente, das 69 universidades que desenvolvem pesquisas sobre educomunicação, 46 o fazem (exclusivamente ou não) em programas das áreas de educação e/ou ensino, o que corresponde a dois terços do total. Ainda, das 35 universidades com mais de uma produção presentes neste trabalho, apenas sete não possuem nenhuma produção nas áreas citadas.

Como exposto no decorrer deste estudo, as teses e dissertações encontradas eram provenientes de programas de pós-graduação em áreas muito diversas, o que é coerente com o caráter intrinsecamente interdisciplinar da educomunicação. ${ }^{21}$ Dessa forma, o fato de mais de $40 \%$ da produção ser realizada em programas voltados à educação e ao ensino demonstra a relevância dessas áreas para consolidar a educomunicação, uma vez que uma fatia significativa da produção acadêmica relacionada ao campo é proveniente delas.

Esses fatores indicam que conhecer a produção aqui relatada é fundamental para conhecer o todo da produção acadêmica brasileira relacionada à educomunicação. Ainda que teses e dissertações nas áreas de educação e ensino sejam, em geral, voltadas mais para a inserção da educomunicação na prática do que para uma reflexão epistemológica acerca do campo, isso não diminui seu valor para a consolidação deste. Afinal, a educomunicação se faz na relação entre teoria e prática. Soares afirma que "uma das tantas singularidades da educomunicação é que ela se constitui justamente das relações múltiplas que propicia"22. Dessa forma, pesquisas que buscam identificar, descrever e analisar as diversas manifestações da educomunicação a partir de suas práticas podem contribuir para o desenvolvimento e a consolidação do campo.

SOARES, Donizete.

Educomunicação: o que é isto?. Gens Instituto de Educação e Cultura, p. 1-12, São Paulo, 2006, p. 4. Disponível em: <http:// www.portalgens.com.br/ baixararquivos/textos/ educomunicacao_o_ que_e_isto.pdf>. Acesso em: 10 dez. 2016.

\section{REFERÊNCIAS BIBLIOGRÁFICAS}

AGUADED, Ignacio. Precisamos de uma revolução educomunicativa para transformar o mundo. Comunicação \& Educação, São Paulo, ano 21, n. 2, jul./dez. 2016. 
BERNARDO, Wanderley Marques; NOBRE, Moacyr Roberto Cuce; JATENE, Fábio Biscegli. A prática clínica baseada em evidências. Parte II - buscando as evidências em fontes de informação. Revista da Associação Médica Brasileira, São Paulo, v. 50, n. 1, p. 104-108, 2004.

BOTELHO, Larissa Lira Roedel; CUNHA, Cristiano Castro de Almeida; MACEDO, Marcelo. O método da revisão integrativa nos estudos organizacionais. Gestão e sociedade, Belo Horizonte, v. 5, n. 11, p. 121-136, maio/ago. 2011.

COOK, Debora J.; MULROW, Cynthia D.; RAYNES, R. Brian. Systematic reviews: synthesis of best evidence for clinical decisions. Annals of Internal Medicine, Philadelphia, v. 126, n. 5, p. 376-380, mar. 1997.

CORNÉLIO, Camila Gallindo. Educomunicação na escola, faz sentido?: projetos educomunicativos de uma escola de referência em ensino médio a partir do que falam os estudantes. Dissertação (mestrado em educação), Universidade Federal de Pernambuco, Pernambuco, 2016.

HOPPE, Bárbara Chiodini Axt. Práticas educomunicativas no contexto escolar: obstáculos e perspectivas. Dissertação (mestrado em extensão rural), Universidade Federal de Santa Maria, Rio Grande do Sul, 2016.

MÁRQUES, Fernanda Telles; TALARICO, Blueth Sabrina Lobo Uchôa. Da comunicação popular à educomunicação: reflexões no campo da "educação como cultura”. Atos de Pesquisa em Educação, Blumenau, v. 11, n. 2, p. $422-$ 443, ago./nov. 2016.

MENDES, Karina Dall Sasso; SILVEIRA, Renata Cristina de Campos Pereira; GALVÃO, Cristina Maria. Revisão integrativa: método de pesquisa para a incorporação de evidências na saúde e na enfermagem. Texto \& Contexto Enfermagem, Florianópolis, v. 17, n. 4, p. 758-764, out./dez. 2008.

OROZCO GÓMEZ, Guillermo. Entre telas: novos papéis comunicativos e educativos dos cidadãos. In: APARICI, Roberto (Org.). Educomunicação: para além do 2.0. São Paulo: Paulinas, 2014.

ROTHER, Edna Terezinha. Revisão sistemática X revisão narrativa. Acta Paulista de Enfermagem, São Paulo, v. 20, n. 2, p. 5-6, abr./jun. 2007.

SAMPAIO, R. F.; MANCINI, M. C. Estudos de revisão sistemática: um guia para síntese criteriosa da evidência científica. Revista Brasileira de Fisioterapia, São Carlos, v. 11, n. 1, p. 83-89, jan./fev. 2007.

SARTORI, Ademilde Silveira. Educomunicação e sua relação com a escola: a promoção de ecossistemas comunicativos e a aprendizagem distraída. Comunicação, mídia e consumo, São Paulo, v. 7, n. 19, p. 33-48, 2010. 
SOARES, Cassia Baldini et al. Revisão integrativa: conceitos e métodos utilizados na enfermagem. Revista da Escola de Enfermagem da USP, São Paulo, v. 48, n. 2, p. 335-345, 2014.

SOARES, Donizete. Educomunicação: o que é isto?. Gens Instituto de Educação e Cultura, São Paulo, p. 1-12, 2006. Disponível em: <http://www. portalgens.com.br/baixararquivos/textos/educomunicacao_o_que_e_isto. pdf>. Acesso em: $10 \mathrm{dez} .2016$.

SOUZA, Marcela Tavares; SILVA, Michelly Dias; CARVALHO, Rachel. Revisão integrativa: o que é e como fazer. Einstein, v. 8, n. 1, p. 102-106, 2010.

URSI, Elisabeth Silva. Prevenção de lesões de pele no perioperatório: revisão integrativa da literatura. Dissertação (mestrado), Escola de Enfermagem de Ribeirão Preto, Universidade de São Paulo, Ribeirão Preto, 2005.

WHITEMORE, Robin; KNAFL, Kathleen. The integrative review: updated methodology. Journal of Advanced Nursing. v. 52, n. 5, p. 546-553, 2005. 